\title{
Beam-Steering Nanophotonic Phased-Array Neural Probes
}

\author{
Wesley D. Sacher ${ }^{1}$, Xinyu Liu ${ }^{1 *}$, Fu-Der Chen ${ }^{2 *}$, Homeira Moradi-Chameh ${ }^{3^{*}}$, Ilan Felts Almog ${ }^{2}$, Thomas \\ Lordello $^{2}$, Michael Chang ${ }^{3}$, Azadeh Naderian ${ }^{3}$, Trevor M. Fowler ${ }^{1}$, Eran Segev ${ }^{1}$, Tianyuan Xue ${ }^{2}$, Sara \\ Mahallati $^{3}$, Taufik A. Valiante ${ }^{3,4,5,2}$, Laurent C. Moreaux ${ }^{1}$, Joyce K. S. Poon ${ }^{2,6}$, Michael L. Roukes ${ }^{1}$ \\ ${ }^{1}$ Division of Physics, Mathematics, and Astronomy, California Institute of Technology, Pasadena, California 91125, USA \\ ${ }^{2}$ Department of Electrical and Computer Engineering, University of Toronto, 10 King's College Rd., Toronto, Ontario M5S 3G4, Canada \\ ${ }^{3}$ Division of Fundamental Neurobiology, Krembil Research Institute, Toronto, Ontario, Canada \\ ${ }^{4}$ Division of Neurosurgery, Department of Surgery, University of Toronto, Toronto, Ontario, Canada \\ ${ }^{5}$ Institute of Biomaterials and Biomedical Engineering, University of Toronto, Toronto, Ontario, Canada \\ ${ }^{6}$ Max Planck Institute for Microstructure Physics, Halle, Germany \\ *Equal Contribution \\ roukes@caltech.edu
}

\begin{abstract}
We demonstrate the first implantable nanophotonic neural probes with integrated silicon nitride phased arrays. Coherent beam-steering is achieved in brain tissue by wavelength tuning. Beam profiles, optogenetic stimulation, and functional imaging are validated in vitro. (c) 2019 The Author(s)
\end{abstract}

OCIS codes: (170.0170) Medical optics and biotechnology; (130.0130) Integrated optics

Advances in optogenetics and the genetic manipulation of neurons have engendered robust and efficient actuators and reporters of brain activity [1,2]. When employed with novel silicon probes implanted proximal to neuronal targets, microscale sources of patterned illumination enable interrogation of brain activity at arbitrary tissue depths with cellular resolution, as the fundamental scattering limitations of conventional free-space optics are circumvented. Recently, multiple optical emitters have been integrated onto silicon (Si) implantable probes using micro- and nano-scale photonic waveguides [3-6] as well as $\mu$ LEDs [7]. Patterned illumination has also been achieved by spatial demultiplexing of tapered multimode fiber implants [8]. Compared to $\mu$ LEDs, passive waveguide-based probes greatly reduce deleterious heat generation in tissue, tailor optical emission profiles more precisely, and are compatible with wafer-scale foundry manufacturing en masse. In comparison to implanted multimode fibers, nanophotonic waveguides enable finer patterned light via their higher spatial resolution and lightsource-array densities. Further, waveguide-based probes utilizing grating-coupler (GC) light emitters demonstrate low-divergence beams in brain tissue over distances of 200-300 $\mu \mathrm{m}[3,4]$. GC arrays can be efficiently addressed using wavelength division multiplexing [3], spatial division multiplexing [4,9], or thermal modulation via on-chip switch matrices [6]. (The latter, however, can reduce thermal benefits over $\mu$ LED-based paradigms.)

Here, we report the first implantable optical beam-steering neural probes employing silicon nitride (SiN) optical phased arrays (OPAs) as microscale light emitters to replace solitary GCs. Their coherently-formed light beams are steerable by input-wavelength tuning. Probe prototypes are fabricated on 4" $\mathrm{Si}$ wafers; operation is validated in vitro in VGAT-ChR2-EYFP and Thy1-GCaMP6s mouse brain slices. The emitters provide sufficient power for optogenetic stimulation and functional imaging.

The OPA neural probes are shown in Fig. 1; they consist of 4 shanks on a $250 \mu \mathrm{m}$ pitch, each $\sim 18 \mu \mathrm{m}$ thick, $3 \mathrm{~mm}$ long, and $50 \mu \mathrm{m}$ wide. A thicker handle region at the probe head facilitates their handling. These probes were fabricated by methods described in [3], and employ photonic circuitry utilizing 200nm thick SiN waveguides. Light
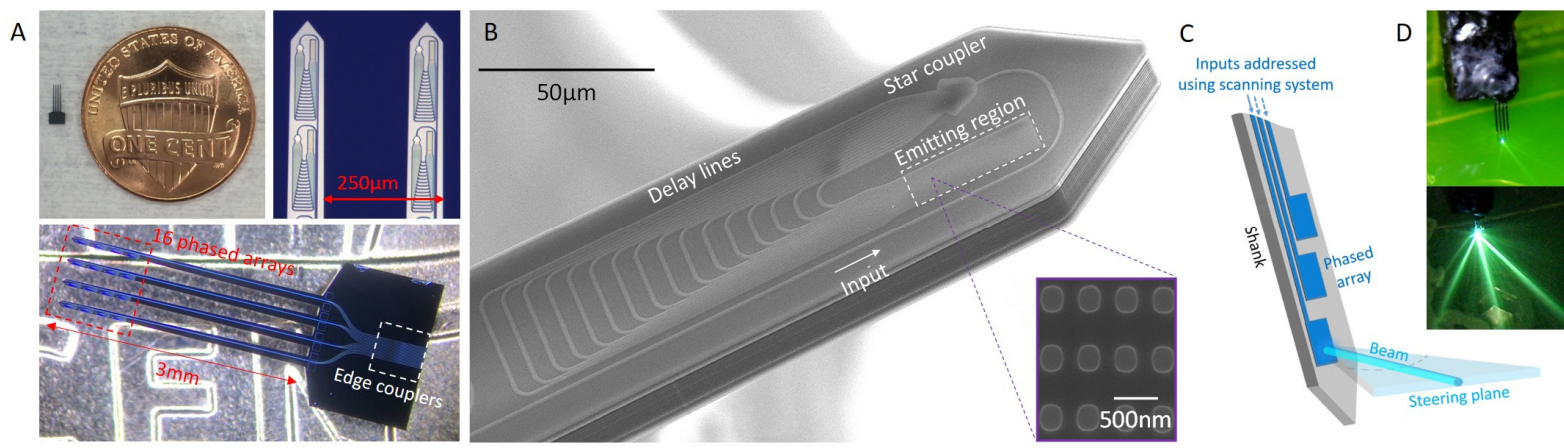

Figure 1. Phased array neural probes. (a) Photograph and microscope images of the neural probe. (b) Annotated scanning electron micrograph (SEM) of a neural probe shank with OPA emitters; the inset is an SEM performed prior to top cladding deposition showing multiple periods of the emitting region grating. The input waveguide is split into 16 paths with uniform length delays between them; each waveguide is terminated by a long, narrow grating, forming an individual emitter in the array. (c) Conceptual diagram of the OPA neural probe operation. (d) Photographs of the packaged probe dipped into fluorescein showing the beam profile at a wavelength of $485 \mathrm{~nm}$. 

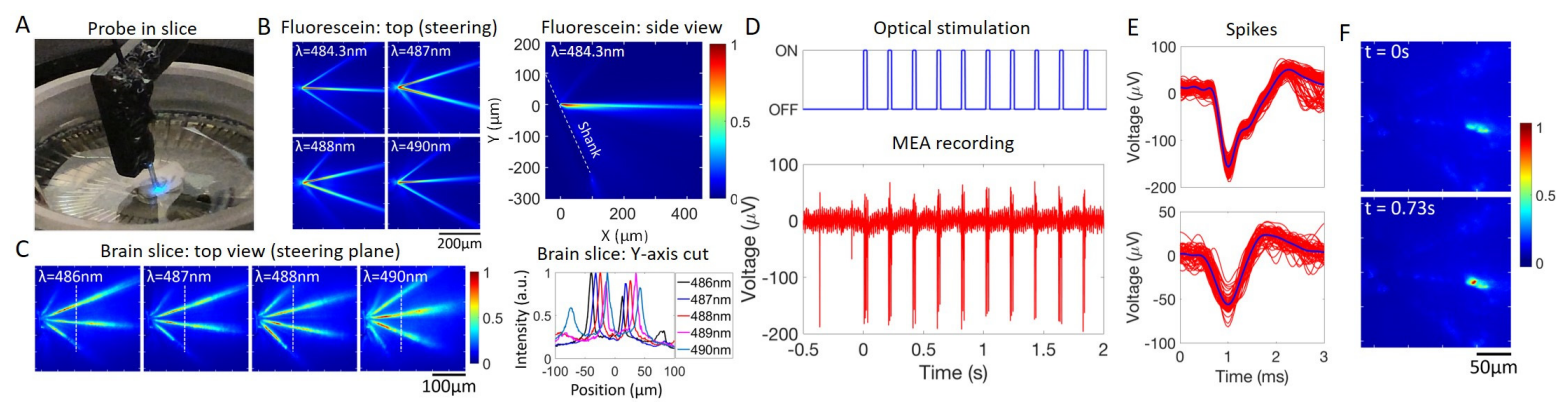

Figure 2. Beam profile measurements and probe validation in brain slices. (a) Photograph of the probe inserted into a brain slice on a microelectrode array (MEA). (b) Top-down (steering plane) and side microscope images of the OPA intensity profile in fluorescein at various

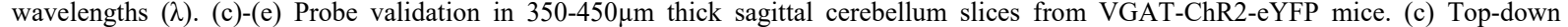
micrographs of the YFP fluorescence excited by the OPA beams at various wavelengths showing beam-steering in brain tissue. Y-axis cut of the micrographs (vertical dashed lines) at a propagation distance of about $105 \mu \mathrm{m}$. (d) Optical stimulus from the probe and corresponding MEA recording on a pad in close proximity to the OPA. (e) Two examples of spike waveforms obtained from the MEA recording following spike sorting. (f) Probe test in a Thy1-GCaMP6s mouse hippocampus slice. Top-down fluorescence images at times $\mathrm{t}=0 \mathrm{~s}$ and $\mathrm{t}=0.73 \mathrm{~s}(25 \mathrm{~ms}$ exposure time) showing high contrast time-dependent fluorescence. All experimental procedures described in this study were reviewed and approved by the University Health Network Animal Care Committee in accordance with the guidelines of Canadian Council on Animal Care.

is coupled onto the chip from a fiber image bundle using on-chip edge couplers. An array of on-chip waveguides subsequently routes light to a set of 16 independent OPAs on the shanks, each capable of generating steered microscale beams. A MEMS mirror-based scanning system similar to that of Ref. [9], couples light to individual cores of the image bundle to controllably address each of the 16 OPAs. Our OPA design is adapted from the infrared silicon waveguide design in Ref. [10] to realize SiN waveguides operating at visible wavelengths required for optogenetics. Delay lines lengths are chosen to achieve a free spectral range (FSR) of $\sim 6 \mathrm{~nm}$, which matches the 484.3-491nm wavelength-tuning range of our external cavity diode laser. The OPA footprint can be significantly reduced further for larger FSRs. Initial characterization, described below, focuses on operating just one of the probe's OPAs; however, we can address multiple OPAs with $\sim 10 \mathrm{~ms}$ access times with our MEMS-scanning system.

To measure beam profiles, the probe is immersed in a fluorescein solution (Fig. 1d) oriented such that the steering plane is parallel to the surface of the fluid. Figure $2 \mathrm{~b}$ shows top-down microscope images of the coherent beams at various wavelengths. A continuous steering range of $\pm 15^{\circ}$ is demonstrated as the wavelength is tuned from $485 \mathrm{~nm}$ to $491 \mathrm{~nm}$; narrow beams are formed that traverse distances of $\sim 400 \mu \mathrm{m}$. The side-view micrograph in Fig. $2 \mathrm{~b}$ confirm the beam's full-width-at-half-maximum (FWHM) is $<18 \mu \mathrm{m}$ over a $400 \mu \mathrm{m}$ propagation distance. Both the angle between additional lobes from higher diffraction orders and the beam extinction can be increased by reducing the pitch between emitters in the OPA (vertical pitch in Fig. 1b inset); however, increasing optical crosstalk within the OPA must be engineered; in this first proof-of-concept demonstration our chosen spacing minimizes such crosstalk. Subsequent designs with optimized array pitch, increased SiN thickness for higher optical confinement and lower crosstalk, and apodization [11] may greatly reduce or eliminate these additional lobes.

The probe was inserted into perfused cerebellum brain slices from 50-60 day old VGAT-ChR2-eYFP mice [12] placed on a microelectrode array for electrophysiological recording. In Fig. 2c, micrographs of YFP fluorescence excited by the steered beams confirm beam-forming and steering by the OPA in scattering brain tissue. Robust spiking with pulsed light from the probe (Fig. 2d, ChR2 excitation) indicates the optical intensity is sufficient for optogenetic stimulation. Additional experiments with brain slices from Thy1-GCaMP6s mice [13] show probes applied to functional imaging. Fig. $2 \mathrm{f}$ shows spontaneous time-dependent fluorescence from a neuron, or small cluster, detected with high contrast using excitation from the main lobe of the OPA. The rise and half decay times of the event are $\approx 0.8 \mathrm{~s}$ and $1.7 \mathrm{~s}$, respectively, consistent with GCaMP6s dynamics. When combined with implantable photodetector arrays, reconfigurable patterned illumination from OPA neural probes will enable implantable lensless imaging systems for deep brain activity mapping beyond the optical scattering limit of free-space imaging.

In conclusion, we have presented implantable optical beam-steering neural probes using OPAs. Coherent beam forming, optogenetic stimulation, and GCaMP6s excitation are achieved in vitro. In vivo experiments are ongoing.

\section{References}

[1] K. Deisseroth, Nat. Neurosci., 18(9), 1213-1225 (2015).

[2] T.-W Chen, et al., Nature 499(7458), 295-300 (2013).

[3] E. Segev, et al., Neurophotonics 4(1), 011002 (2017).

[4] S. Libbrecht, et al., J. Neurophysiol. 120(1), 149-161 (2018).

[5] A. Mohanty et al., in Conference on Lasers and Electro-Optics, (Optical Society of America, 2018), paper ATh3Q.1.

[6] K. Kampasi, et al., Scientific Reports, 6, 30961 (2016).

[7] F. Wu et al., Neuron, 88(6), 1136-1148 (2015).

[8] F. Pisanello, et al., Neuron, 82(6), 1245-1254 (2014).

[9] A. N. Zorzos, et al., Opt. Lett. 37(23), 4841-4843 (2012).

[10] K. Van Acoleyen et al., IEEE Photon. Technol. Lett. 23(17), 1270-1272 (2011).

[11] D. N. Hutchison, et al., Optica 3(8), 887-890 (2016).

[12] S. Zhao, et al., Nat. Methods, 8(9), 745-752 (2011).

[13] H. Dana, et al., PLoS One, 9(9), e108697 (2014). 